\title{
Symmetric periodic orbits near a heteroclinic loop formed by two singular points and their invariant manifolds of dimension 1 and 2
}

\author{
Montserrat Corbera ${ }^{(1)}$, Jaume Llibre $^{(2)}$ and Ernesto \\ Pérez-Chavela ${ }^{(3)}$ \\ (1) Departament de Tecnologies digitals i de la Informació, \\ Laura 13, 08500 Vic, Barcelona, Spain. \\ (2) Departament de Matemàtiques, Universitat Autònoma de Barcelona, 08193 - \\ Bellaterra, Barcelona, Spain. \\ (3) Departamento de Matemáticas, Universidad Autónoma Metropolitana-I, Apdo. \\ Postal 55-534, 09340 México D.F., México. \\ E-mail: montserrat.corbera@uvic.cat, jllibre@mat.uab.cat, epc@xanum.uam.mx
}

\begin{abstract}
In this paper we consider vector fields in $\mathbb{R}^{3}$ that are invariant under a suitable symmetry and that posses a "generalized heteroclinic loop" $\mathcal{L}$ formed by two singular points $\left(e^{+}\right.$and $\left.e^{-}\right)$and their invariant manifolds: one of dimension 2 (a sphere minus the points $e^{+}$and $e^{-}$) and one of dimension 1 (the open diameter of the sphere having endpoints $e^{+}$and $e^{-}$). In particular, we analyze the dynamics of the vector field near the heteroclinic loop $\mathcal{L}$ by means of a convenient Poincaré map, and we prove the existence of infinitely many symmetric periodic orbits near $\mathcal{L}$. We also study two families of vector fields satisfying this dynamics. The first one is a class of quadratic polynomial vector fields in $\mathbb{R}^{3}$, and the second one is the charged rhomboidal four body problem.
\end{abstract}

Key words and phrases: Heteroclinic loop, symmetric periodic orbits, polynomial vector fields, charged rhomboidal four body problem, collision manifold.

(2000) AMS Mathematics Subject Classification: 70F10, 78A35, 34C25. 


\section{Introduction}

In this paper we study the periodic motion around a generalized heteroclinic loop $\mathcal{L}$ formed by a 2-dimensional sphere $\mathbb{S}^{2}$ and an interior diameter $\Gamma$ of the sphere, see Fig. 1. We suppose that the flow of a system $X$ having such a loop is defined on the closed ball $\mathbb{D}^{3}$ of $\mathbb{R}^{3}$ having as boundary $\mathbb{S}^{2}$. On $\mathbb{S}^{2}$ we have two foci, $e^{+}$and $e^{-}$, diametrally opposite at the endpoints of the diameter $\Gamma$. Every orbit on $\mathbb{S}^{2}$ different from the two foci starts spiraling at $e^{-}$and ends spiraling at $e^{+}$. In fact, $\mathbb{S}^{2} \backslash\left\{e^{+}, e^{-}\right\}$ is the 2-dimensional unstable manifold of $e^{-}$which coincides with the 2 -dimensional stable manifold of $e^{+}$. Moreover, the diameter $\Gamma$ is formed by a unique orbit starting at $e^{+}$and ending at $e^{-}$; i.e. $\Gamma$ is the 1 -dimensional unstable manifold of $e^{+}$which coincides with the 1 -dimensional stable manifold of $e^{-}$. We assume that the flow on $\mathbb{D}^{3}$ is symmetric with respect to a line of symmetry $L$ orthogonal to the diameter $\Gamma$.

Analyzing the dynamics of the vector field $X$ near this generalized heteroclinic loop $\mathcal{L}$ by means of a convenient Poincaré map and using the symmetry of the problem we can prove the existence of infinitely many symmetric periodic orbits of $X$ near $\mathcal{L}$. The key point of this paper is that we can obtain the properties of the Poincaré map that are necessary to prove the existence of symmetric periodic orbits by using geometric arguments instead of using the analytic expression of the Poincaré map. This avoids in this case the usual hard computations necessary for computing the image of the Poincaré map and its intersection with the line of symmetry. Moreover it allows us to prove the existence of infinitely many periodic orbits for any vector field $\mathrm{X}$ possessing the heteroclinic loop $\mathcal{L}$ and the mentioned symmetry although we do not know the explicit analytic expression of the vector field. Other papers following these geometric approach are $[2,3,4,6]$.

To use heteroclinic loops for finding periodic orbits near using the geometry close to these loops more than the tedious computations associated to the analytic expressions of the Poincaré maps has a long tradition in the qualitative study of the differential systems, see for instance $[2,4,6,8,10,18]$. In many cases the heteroclinic loops are formed by stable and unstable manifolds of the same dimension (see $[12,13]$ ), or by heteroclinic orbits which are intersection of stable and unstable manifolds (see $[6,7,10]$ ). The heteroclinic loop $\mathcal{L}$ studied here is special in the sense that it is formed by stable and unstable manifolds of different dimension, and their orbits are not intersection of stable and unstable manifolds. As far as we know, the use of these kind of heteroclinic loops in order to find periodic orbits is not very common in the literature and nevertheless it is a very interesting problem from a dynamical point of view because it exhibits the complicated dynamics near these heteroclinic loops in a very simple form. In [4] the authors use similar techniques than the ones used here for proving the existence of periodic orbits for a particular case of the charged rhomboidal 4-body problem, but they do not mention explicitly the existence of the heteroclinic loop. Here we treat the problem in a more general form, we describe the heteroclinic loop $\mathcal{L}$, we give the conditions that must verify a vector field $X$ in $\mathbb{D}^{3}$ in order to have periodic orbits near 


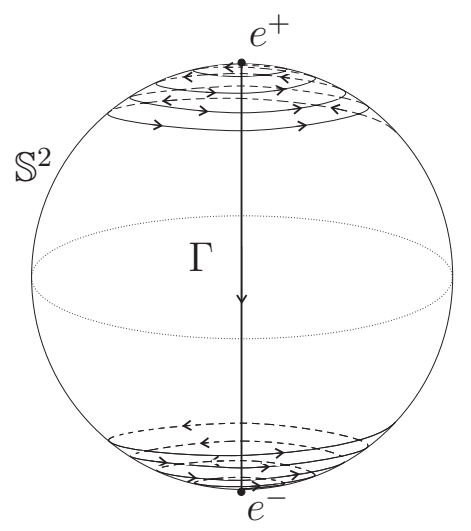

Figure 1. The generalized heteroclinic loop.

the heteroclinic loop $\mathcal{L}$, and finally we give two particular examples of vector fields satisfying this dynamics.

In Section 2 we prove the existence of infinitely many periodic orbits for any vector field defined on the closed ball $\mathbb{D}^{3}$ having the described generalized heteroclinic loop and the mentioned symmetry. In Section 3 we apply the analysis done in Section 2 to a class of quadratic polynomial vector fields in $\mathbb{R}^{3}$, showing that the easiest nonlinear systems already present complicated dynamics. Finally in Section 4 we use the results of Section 2 to prove the existence of infinitely many symmetric periodic orbits for the charged rhomboidal 4-body problem that pass near total collision, extending results of [4]. The charged rhomboidal 4-body problem consist of describing the dynamics of 4 point particles endowed with a positive mass and an electrostatic charge of any sign, moving under the influence of the respective Newtonian and Coulombian forces in such a way that the four particles form a rhombus at every time.

\section{The main Theorem}

Without loss of generality we can assume that the closed ball is $\mathbb{D}^{3}=\left\{(x, y, z) \in \mathbb{R}^{3}\right.$ : $\left.x^{2}+y^{2}+z^{2} \leqslant 1\right\}$, its boundary is $\mathbb{S}^{2}=\left\{(x, y, z) \in \mathbb{R}^{3}: x^{2}+y^{2}+z^{2}=1\right\}$, the interior diameter is $\Gamma=\left\{(x, y, z) \in \mathbb{R}^{3}: x=y=0,-1<z<1\right\}$ and the line of symmetry is $L=\left\{(x, y, z) \in \mathbb{R}^{3}: x=z=0\right\}$. Assume that the vector field $X=(f(x, y, z), g(x, y, z), h(x, y, z))$ is defined on the closed ball $\mathbb{D}^{3}$ and it satisfies the following conditions:

$\left(C_{1}\right)$ The sphere $\mathbb{S}^{2}$ is invariant under the flow of $X$.

$\left(C_{2}\right)$ On $\mathbb{S}^{2}$ the vector field $X$ has two foci, $e^{+}=\{(0,0,1)\}$ and $e^{-}=\{(0,0,-1)\}$.

$\left(C_{3}\right)$ Every orbit on $\mathbb{S}^{2}$ different from the two foci starts spiraling at $e^{-}$and ends spiraling at $e^{+}$. In fact, $\mathbb{S}^{2} \backslash\left\{e^{+}, e^{-}\right\}$is the 2-dimensional unstable manifold of $e^{-}\left(W_{e^{-}}^{u}\right)$ which coincides with the 2-dimensional stable manifold of $e^{+}\left(W_{e^{+}}^{s}\right)$.

$\left(C_{4}\right)$ The diameter $\Gamma$ is formed by a unique orbit starting at $e^{+}$and ending at $e^{-}$; i.e. $\Gamma$ is the 1 -dimensional unstable manifold of $e^{+}\left(W_{e^{+}}^{u}\right)$ which coincides with the 
1-dimensional stable manifold of $e^{-}\left(W_{e^{-}}^{s}\right)$.

$\left(C_{5}\right)$ The flow on $\mathbb{D}^{3}$ is invariant under the time-reversibility symmetry $(x, y, z, t) \longrightarrow$ $(-x, y,-z,-t)$; that is, it is symmetric with respect to the line of symmetry $L$ (i.e. the $y$-axis) and a change of the sign of the time.

Under these assumptions the vector field $X$ possesses a generalized heteroclinic loop $\mathcal{L}$ formed by the equilibrium points $e^{+}$and $e^{-}$and the invariant manifolds $W_{e^{-}}^{u}=W_{e^{+}}^{s}$ and $W_{e^{-}}^{s}=W_{e^{+}}^{u}$.

Let $P=\{(0,0,0)\}$ be the intersection point of the line of symmetry $L$ with the diameter $\Gamma$ and let $Q=\{(0,1,0)\}$ and $R=\{(0,-1,0)\}$ be the intersection points of $L$ with $\mathbb{S}^{2}$.

Proposition 1 Assume that the vector field $X$ is defined on the closed ball $\mathbb{D}^{3}$ and it satisfies conditions $\left(C_{1}\right)-\left(C_{5}\right)$, then $X$ has infinitely many periodic orbits near the heteroclinic loop $\mathcal{L}$ that cross exactly 2 times the plane $z=0$ during a period. In particular, we have infinitely many periodic orbits with one crossing near the point $P$ and the other one near $Q$, and infinitely many periodic orbits with one crossing near $P$ and the other one near $R$, see Figure 2.

Proof: Using the invariance of the vector field $X$ with respect to the symmetry $(x, y, z, t) \longrightarrow(-x, y,-z,-t)$ we have that if $\phi(t)=(x(t), y(t), z(t))$ is a solution of $X$, then $\psi(t)=(-x(-t), y(-t),-z(-t))$ is also a solution. This symmetry can be used in the standard way in order to obtain symmetric periodic solutions. Using the symmetry and the uniqueness theorem on the solutions of the differential system associated to $X$ it is easy to see that if $x(0)=z(0)=0$, then the orbits $\phi$ and $\psi$ must be the same. Moreover, if there exists a time $\tau>0$ such that $x(\tau)=z(\tau)=0$ and $x(\tau)^{2}+z(\tau)^{2} \neq 0$ for all $0<t<\tau$, then the orbit must be periodic of period $2 \tau$. In other words, if an orbit intersects the line of symmetry $L$ in two different points, then it is a periodic orbit. The use of time-reversibility symmetries in order to find symmetric periodic orbits is a classical technique (see [17]) and it is very used at the present time (see for instance [14]).

We start giving some definitions and some notations. Assume that $\varepsilon_{i}>0$ are sufficiently small values for all $i=1,2,3$. We consider the segment $\gamma=\{(0, y, 0) \in L$ : $\left.y \in\left(0, \varepsilon_{1}\right)\right\}$, and the section $\Sigma=\left\{(x, y, z) \in \mathbb{D}^{3}: z=0\right\}$. We also consider a small topological cylinder in a neighbourhood of the equilibrium point $e^{-}=\{(0,0,-1)\}$ with base on $\mathbb{S}^{2}$ and boundaries $\Sigma_{1}$ and $\Sigma_{2}$ with $\Sigma_{1}=\left\{(x, y, z) \in \mathbb{D}^{3}: z=-1+\varepsilon_{2}, x^{2}+y^{2} \leqslant\right.$ $\left.\varepsilon_{3}\right\}$ and $\Sigma_{2}=\left\{(x, y, z) \in \mathbb{D}^{3}: z \leqslant-1+\varepsilon_{2}, x^{2}+y^{2}=\varepsilon_{3}\right\}$, see Figure 2 .

We define a map $\pi: \gamma \longrightarrow \Sigma$ in the following way. We denote by $\varphi(t, q)$ the flow generated by system $X$, satisfying $\varphi(0, q)=q$. We consider the diffeomorphism $\pi_{0}: \gamma \rightarrow \Sigma_{1}$ defined by $\pi_{0}(q)=p$, where $p$ is the point at which the orbit $\varphi(t, q)$ intersects the cross section $\Sigma_{1}$ for the first time. By the continuity of the flow $\varphi$ with respect to initial conditions, if $q$ is sufficiently close to the point $P$, then the orbit $\varphi(t, q)$ is close to the orbit $\Gamma$ for all $t$ in a finite interval of time. Since the orbit $\Gamma$ expends a 


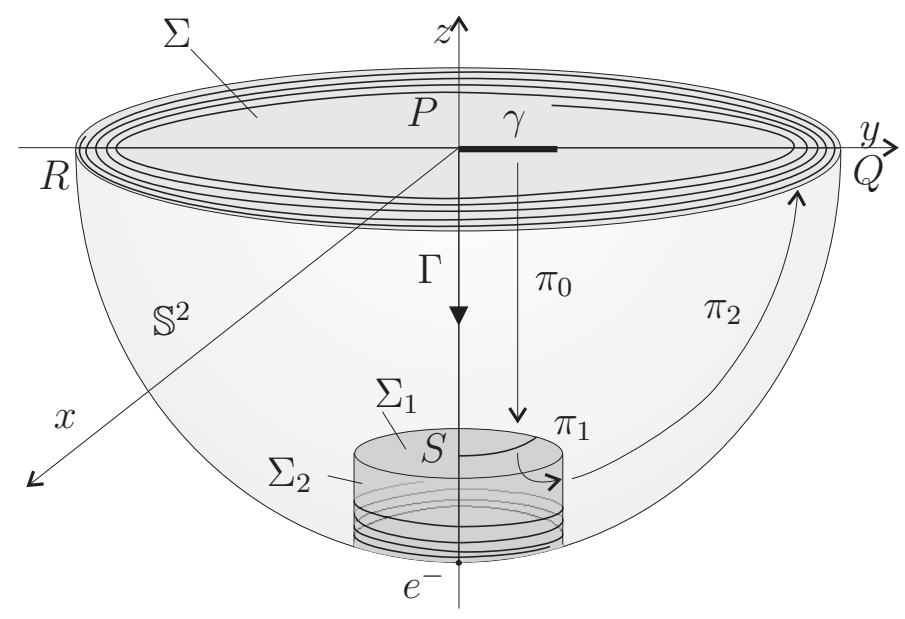

Figure 2. The map $\pi$.

finite time for going from the point $P$ to the point $S=\Sigma_{1} \cap \Gamma$, we can guarantee that for all $q \in \gamma$ sufficiently close to $P$ the orbit $\varphi(t, q)$ intersects $\Sigma_{1}$. Consequently if $\varepsilon_{1}$ is sufficiently small, then the map $\pi_{0}$ is well defined. Moreover, the image by $\pi_{0}$ of $\gamma$ is an $\operatorname{arc}$ on $\Sigma_{1}$ with $S$ as one of its endpoints (see Figure 2).

We consider a second diffeomorphism $\pi_{1}: \Sigma_{1} \longrightarrow \Sigma_{2}$ defined by $\pi_{1}(q)=p$, where $p$ is the point at which the orbit $\varphi(t, q)$ intersects $\Sigma_{2}$ for the first time. If $\varepsilon_{3}$ is sufficiently small, then the orbit $\varphi(t, q)$ intersects $\Sigma_{2}$ for all $q \in \Sigma_{1} \backslash\{S\}$, because $e^{-}$is a hyperbolic equilibrium point with $W_{e^{-}}^{u}=\mathbb{S}^{2} \backslash\left\{e^{+}, e^{-}\right\}$and $W_{e^{-}}^{s}=\Gamma$. Moreover, since $e^{-}$is an unstable focus on $\mathbb{S}^{2}$ and the point $\pi_{0}(P)=S \in W_{e^{-}}^{s}$, the image $\pi_{1}\left(\pi_{0}(\gamma)\right)$ is a spiral on $\Sigma_{2}$ that approaches to $\mathbb{S}^{2}$, when we approach to $P$, spiraling infinitely many times (see again Figure 2).

We define a third map $\pi_{2}: \Sigma_{2} \longrightarrow \Sigma$, defined by $\pi_{2}(q)=p$, where $p$ is the point at which the orbit $\varphi(t, q)$ intersects $\Sigma$ for the first time. Since from condition $\left(C_{3}\right)$ every orbit on $\mathbb{S}^{2}$ starts at $e^{-}$and ends at $e^{+}$, if $\varepsilon_{2}$ and $\varepsilon_{3}$ are sufficiently small then the point $p$ is well defined.

Finally, we consider the map $\pi: \gamma \longrightarrow \Sigma$ defined by $\pi=\pi_{2} \circ \pi_{1} \circ \pi_{0}$. Since the orbits expend a finite time for going from $\Sigma_{2}$ to $\Sigma, \pi_{2}$ is a diffeomorphism. Therefore the image $\pi(\gamma)$ is a spiral on $\Sigma$ that approaches to $\mathbb{S}^{2}$, when we approach to $P$, spiraling infinitely many times.

We note that $\pi(\gamma)$ intersects the line of symmetry $L$ infinitely many times near the point $Q$, and infinitely many times near the point $R$. Since the points of $\gamma$ belong to the line of symmetry, those intersection points correspond to orbits of $X$ that cross the line of symmetry at two different points; that is, they correspond to symmetric periodic orbits. By the construction, these periodic orbits cross exactly 2 times the plane $z=0$.

The periodic orbits given by Proposition 1 are obtained from the intersection points of the image by $\pi$ of the segment $\gamma=\left\{(0, y, 0) \in L: y \in\left(0, \varepsilon_{1}\right)\right\}$ with the line of 


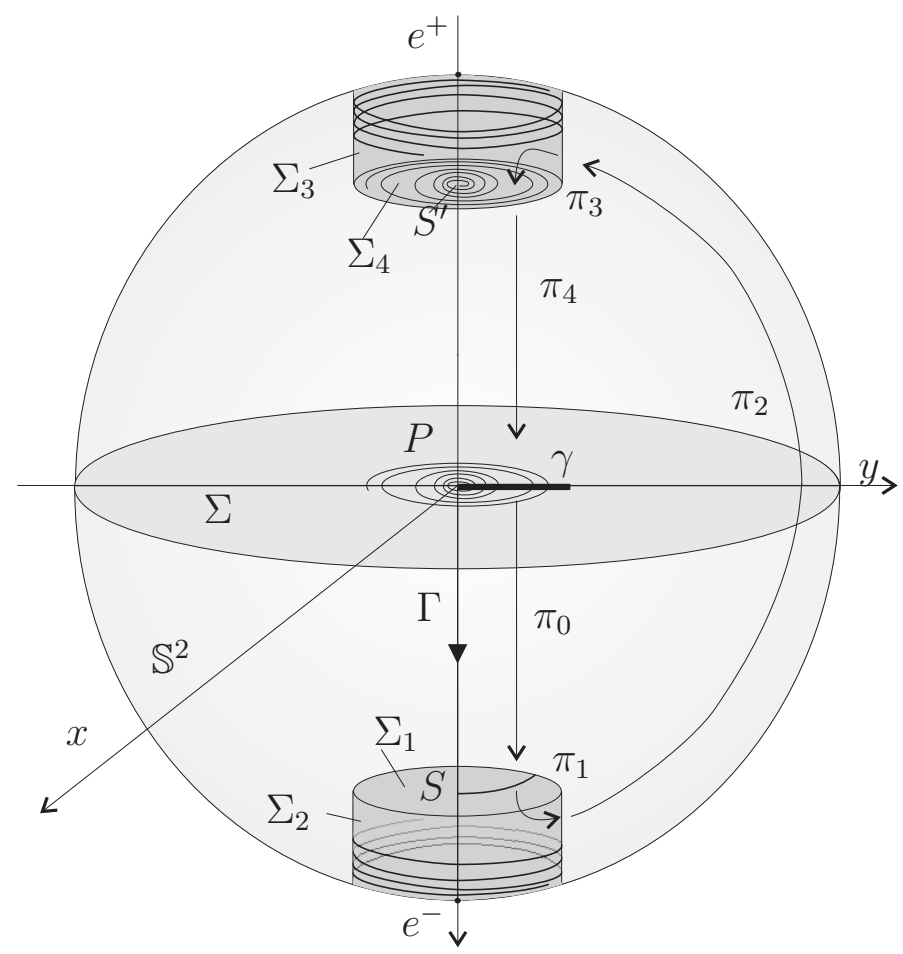

Figure 3. The map $\Pi$.

symmetry $L$. If we repeat the arguments of the proof of Proposition 1 with the segment $\gamma^{\prime}=\left\{(0, y, 0) \in L: y \in\left(-\varepsilon_{1}, 0\right)\right\}$ instead of $\gamma$ we would obtain infinitely many symmetric periodic orbits that are different from the ones obtained above.

Proposition 2 Assume that the vector field $X$ is defined on the closed ball $\mathbb{D}^{3}$ and it satisfies conditions $\left(C_{1}\right)-\left(C_{5}\right)$, then $X$ has infinitely many periodic orbits near the heteroclinic loop $\mathcal{L}$ that cross exactly 4 times the plane $z=0$ during a period.

Proof: We proceed in a similar way as in the proof of Proposition 1. We consider $\gamma, \Sigma, \Sigma_{1}$ and $\Sigma_{2}$ defined as in that proof; and we consider another small topological cylinder in a neighbourhood of the equilibrium point $e^{+}=\{(0,0,1)\}$ with base on $\mathbb{S}^{2}$ and boundaries $\Sigma_{3}$ and $\Sigma_{4}$ where $\Sigma_{3}=\left\{(x, y, z) \in \mathbb{D}^{3}: z \geqslant 1-\varepsilon_{4}, x^{2}+y^{2}=\varepsilon_{5}\right\}$, $\Sigma_{4}=\left\{(x, y, z) \in \mathbb{D}^{3}: z=1-\varepsilon_{4}, x^{2}+y^{2} \leqslant \varepsilon_{5}\right\}$, and $\varepsilon_{4}, \varepsilon_{5}>0$ are sufficiently small (see Figure 3).

Let $\pi_{0}$ and $\pi_{1}$ be defined as in the proof of Proposition 1. We define a third map $\pi_{2}: \Sigma_{2} \longrightarrow \Sigma_{3}$, defined by $\pi_{2}(q)=p$, where $p$ is the point at which the orbit $\varphi(t, q)$ intersects $\Sigma_{3}$ for the first time. Since $W_{e^{-}}^{u}=W_{e^{+}}^{s}=\mathbb{S}^{2} \backslash\left\{e^{+}, e^{-}\right\}$, if $\varepsilon_{2}$ and $\varepsilon_{3}$ are sufficiently small, then the point $p$ is well defined. Moreover, the orbits expend a finite time for going from $\Sigma_{2}$ to $\Sigma_{3}$, so $\pi_{2}$ is a diffeomorphism. Therefore the image $\pi_{2}\left(\pi_{1}\left(\pi_{0}(\gamma)\right)\right)$ is a spiral on $\Sigma_{3}$ that approaches to $\mathbb{S}^{2}$, when we approach to $P$, spiraling infinitely many times (see Figure 3 ).

We define another map $\pi_{3}: \Sigma_{3} \longrightarrow \Sigma_{4}$ defined by $\pi_{3}(q)=p$, where $p$ is the point at which the orbit $\varphi(t, q)$ intersects $\Sigma_{4}$ for the first time. If $\varepsilon_{4}$ is sufficiently small, 
then this point $p$ is well defined because $e^{+}$is a hyperbolic equilibrium point with $W_{e^{+}}^{s}=\mathbb{S}^{2} \backslash\left\{e^{+}, e^{-}\right\}$and $W_{e^{+}}^{u}=\Gamma$. Moreover, the image $\pi_{3}\left(\pi_{2}\left(\pi_{1}\left(\pi_{0}(\gamma)\right)\right)\right)$ is a spiral on $\Sigma_{4}$ that approaches to the point $S^{\prime}=\Sigma_{4} \cap \Gamma$, when we approach to $P$, spiraling infinitely many times (see again Figure 3).

We define $\pi_{4}: \Sigma_{4} \longrightarrow \Sigma$ in a similar way than $\pi_{0}^{-1}$. Finally we consider the map $\Pi: \gamma \longrightarrow \Sigma$ defined by $\Pi=\pi_{4} \circ \pi_{3} \circ \pi_{2} \circ \pi_{1} \circ \pi_{0}$. The image $\Pi(\gamma)$ is a spiral on $\Sigma$ that approaches to $P$ spiraling infinitely many times.

We note that $\Pi(\gamma)$ intersects $\gamma$ and $\gamma^{\prime}$ both infinitely many times. Since the points of $\gamma$ belong to the line of symmetry, those intersection points correspond to symmetric periodic orbits of $X$. The points of $\gamma \cap \Pi(\gamma)$ correspond to the symmetric periodic orbits found in Proposition 1 (that have exactly 2 intersection points with $z=0$ ), and probably to new ones (that have exactly 4 intersection points with $z=0$ ). The points of $\gamma^{\prime} \cap \Pi(\gamma)$ correspond to symmetric periodic orbits that cannot be found in Proposition 1 and that have exactly 4 intersection points with $z=0$.

We can apply similar arguments to analyze the intersection points of $\gamma^{\prime} \cap \Pi\left(\gamma^{\prime}\right)$ and $\gamma \cap \Pi\left(\gamma^{\prime}\right)$. We note that the symmetric periodic orbits obtained form the points of $\gamma^{\prime} \cap \Pi(\gamma)$ and $\gamma \cap \Pi\left(\gamma^{\prime}\right)$ are the same.

Doing similar arguments as the ones used in Propositions 1 and 2, it is not difficult to see that the periodic orbits coming from the intersection points of $\pi(\Pi(\gamma))$ with the line of symmetry $L$ provide the symmetric periodic orbits found in Proposition 1, and additionally provide infinitely many symmetric periodic orbits that cross exactly 6 times the plane $z=0$ during a period. The intersection points of $\Pi^{2}(\gamma)$ with the line of symmetry $L$ provide the symmetric periodic orbits found in Proposition 2, and additionally provide infinitely many symmetric periodic orbits that cross exactly 8 times the plane $z=0$ during a period; and so on. In short we have proved the following result, which is the main result of this paper.

Theorem 3 Assume that the vector field $X$ is defined on the closed ball $\mathbb{D}^{3}$ and it satisfies conditions $\left(C_{1}\right)-\left(C_{5}\right)$. For each $n \in \mathbb{N}$ the vector field $X$ has infinitely many periodic orbits near the heteroclinic loop $\mathcal{L}$ that cross exactly $2 n$ times the plane $z=0$ during a period.

\section{Polynomial vector fields}

In this section we characterize the class of quadratic polynomial vector fields in $\mathbb{R}^{3}$ satisfying conditions $\left(C_{1}\right)-\left(C_{5}\right)$. So the easier nonlinear vector fields in $\mathbb{R}^{3}$ already present a complicated dynamics as the provided by Theorem 3 .

We consider an arbitrary quadratic polynomial vector field $X=(P, Q, R)$ in $\mathbb{R}^{3}$ with

$$
\begin{aligned}
P & =\sum_{0 \leqslant i+j+k \leqslant 2} a_{i j k} x^{i} y^{j} z^{k}, \quad Q=\sum_{0 \leqslant i+j+k \leqslant 2} b_{i j k} x^{i} y^{j} z^{k}, \\
R & =\sum_{0 \leqslant i+j+k \leqslant 2} c_{i j k} x^{i} y^{j} z^{k} .
\end{aligned}
$$


Assuming that the straight line $x=y=0$ is invariant under the flow of $X$ (condition $\left(C_{4}\right)$ ) we have that

$$
a_{000}=a_{001}=a_{002}=b_{000}=b_{001}=b_{002}=0 .
$$

Imposing that the system associated to $X$ is invariant under the symmetry $\left(C_{5}\right)$ we get that

$$
\begin{aligned}
a_{100} & =a_{110}=a_{011}=b_{200}=b_{010}=b_{020}=b_{101}=c_{100} \\
& =c_{110}=c_{001}=c_{011}=0 .
\end{aligned}
$$

Now we impose condition $\left(C_{1}\right)$. The sphere $\mathbb{S}^{2}$ is invariant under the flow of $X$ when the function $2 x \dot{x}+2 y \dot{y}+2 z \dot{z}$ evaluated at $z= \pm \sqrt{1-x^{2}-y^{2}}$ equals to zero. By imposing this condition we obtain the following relations

$$
\begin{array}{llll}
a_{010}=-b_{100}, & a_{020}=-b_{110}, & a_{101}=c_{002}-c_{200}, & a_{200}=0 \\
b_{011}=c_{002}-c_{020}, & c_{000}=-c_{002}, & c_{010}=0, & c_{101}=0 .
\end{array}
$$

Condition $\left(C_{4}\right)$ says that $X$ has no singular points on $\Gamma=\left\{(x, y, z) \in \mathbb{R}^{3}: x=\right.$ $y=0,-1<z<1\}$ and that the flow of $X$ on $\Gamma$ goes in the decreasing direction of the $z$ axis. The flow of $X$ on the straight line $x=y=0$ is given by $\dot{x}=0, \dot{y}=0$ and $\dot{z}=c_{002}\left(z^{2}-1\right)$. So if $c_{002}>0$, then condition $\left(C_{4}\right)$ is satisfied.

From condition $\left(C_{2}\right)$, the equilibrium points $e^{+}=\{(0,0,1)\}$ and $e^{-}=\{(0,0,-1)\}$ must be foci. Let $\alpha=c_{020}+c_{200}-2 c_{002}$ and $\beta=\left(c_{020}-c_{200}\right)^{2}-4 b_{100}{ }^{2}$. The eigenvalues of the linear part of $X$ at the equilibrium points $e^{+}$and $e^{-}$are

$$
\lambda_{1}=2 c_{002}, \quad \lambda_{2,3}=\frac{-\alpha \pm \sqrt{\beta}}{2},
$$

and

$$
\lambda_{1}=-2 c_{002}, \quad \lambda_{2,3}=\frac{\alpha \mp \sqrt{\beta}}{2},
$$

respectively. So the coefficients must satisfy that $\beta<0$, and consequently $\left|b_{100}\right|>$ $\left|c_{020}-c_{200}\right| / 2$.

Finally we impose that every orbit on $\mathbb{S}^{2}$ different from the two foci starts spiraling at $e^{-}$and ends spiraling at $e^{+}$(condition $\left(C_{3}\right)$ ). In order to impose this condition we write the vector field $X$ in spherical coordinates $x=r \cos \theta \cos \phi, y=r \cos \theta \sin \phi$ and $z=r \sin \theta$. If the derivative $d \theta / d t$ evaluated at $r=1$ is positive for all $\theta \in(-\pi / 2, \pi / 2)$ and $\phi \in[0,2 \pi)$, then condition $\left(C_{3}\right)$ is satisfied. After some computations we get

$$
\left.\frac{d \theta}{d t}\right|_{r=1}=\frac{1}{2} \cos \theta\left[\alpha+\left(c_{200}-c_{020}\right) \cos (2 \phi)\right]
$$

So we need that $\alpha>\left|c_{200}-c_{020}\right|$.

In short we have proved the following result.

Proposition 4 The quadratic polynomial vector fields of the form

$$
X=\left(-a y-b y^{2}+(c-d) x z, a x+b x y+(c-e) y z,-c+d x^{2}+e y^{2}+c z^{2}\right)
$$

with $c>0, e+d-2 c>|d-e|$ and $|a|>|e-d| / 2$ satisfy conditions $\left(C_{1}\right)-\left(C_{5}\right)$. 


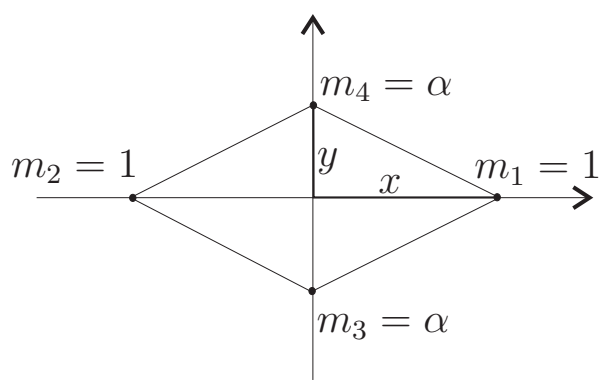

Figure 4. The rhombus formed by the four particles.

Then applying Theorem 3 we obtain the following result.

Theorem 5 Let $X$ be a quadratic vector field defined as in Proposition 4 , and let $\mathcal{L}$ be the heteroclinic loop formed by the equilibrium points $e^{+}=\{(0,0,1)\}$ and $e^{-}=$ $\{(0,0,-1)\}$ and the invariant manifolds $\mathbb{S}^{2}=\left\{(x, y, z) \in \mathbb{R}^{3}: x^{2}+y^{2}+z^{2}=1\right\} \backslash\left\{e^{+}, e^{-}\right\}$ and $\Gamma=\left\{(x, y, z) \in \mathbb{R}^{3}: x=0, y=0,-1<z<1\right\}$. For each $n \in \mathbb{N}$ the vector field $X$ has infinitely many periodic orbits near the heteroclinic loop $\mathcal{L}$ that cross exactly $2 n$ times the plane $z=0$ during a period.

\section{The charged 4-body problem}

\subsection{Introduction}

The charged 4-body problem corresponds to the study of the dynamics of 4 point particles endowed with a positive mass and an electrostatic charge of any sign, moving under the influence of the respective Newtonian and Coulombian forces. These kind of problems have been studied among others in $[1,2,4,16]$. In this paper we study a particular problem, when the point particles form a rhombus at every time. That is, we consider four point particles with masses $m_{1}, m_{2}, m_{3}$ and $m_{4}$ and charges $q_{1}, q_{2}, q_{3}$ and $q_{4}$, located at the vertices of a rhombus. From here on, the center of mass is fixed at the origin. In order to preserve the rhomboidal configuration for all time we must take $m_{1}=m_{2}, m_{3}=m_{4}, q_{1}=q_{2}, q_{3}=q_{4}$ and suitableness symmetrical velocities for the four particles, see Figure 4. Choosing a convenient unit of mass we can suppose that $m_{1}=m_{2}=1$ and $0<m_{3}=m_{4}=\alpha \leqslant 1$.

Taking the units of mass and of charge conveniently we can assume that the gravitational constant and the Coulomb's constant are equal to one. We define the new parameters $\lambda_{i j}=m_{i} m_{j}-q_{i} q_{j}$ for $i, j=1,2,3,4$ and $i \neq j$, then if $\lambda_{i j}>0$ the resultant force between the particles $i$ and $j$ is attractive, and if $\lambda_{i j}<0$ then it is repulsive. It is clear that depending on the sign of the above parameters it is possible to avoid any kind of binary collision or even the total collision.

We will prove that, fixed a level of energy $E_{h}$ with $h<0$, the charged rhomboidal 4body problem satisfies conditions $\left(C_{1}\right)-\left(C_{5}\right)$ and consequently Theorem 3 can be applied to find infinitely many symmetric periodic orbits of this problem. 
In [4] the authors use very similar techniques than the ones used here in order to prove the existence of infinitely many symmetric periodic orbits passing near total collision for the charged rhomboidal four body in the particular case $\lambda_{34}=0$. Here we find those periodic orbits for values of $\lambda_{34}<0$.

\subsection{Equations of motion}

Let $x \geqslant 0$ be the half distance between the particles 1 and 2 and let $y \geqslant 0$ be the half distance between the particles 3 and 4 , see Figure 4 . We observe that $x=0$ corresponds to double collision between the particles 1 and $2 ; y=0$ represents double collision between the particles 3 and 4 . The total collision corresponds to $x=0$ and $y=0$ simultaneously.

In these coordinates the equations of motion can be written as

$$
\ddot{x}=-\left[\frac{\lambda_{12}}{4 x^{2}}+\frac{2 \lambda_{13} x}{\left(x^{2}+y^{2}\right)^{\frac{3}{2}}}\right], \quad \ddot{y}=-\frac{1}{\alpha}\left[\frac{\lambda_{34}}{4 y^{2}}+\frac{2 \lambda_{13} y}{\left(x^{2}+y^{2}\right)^{\frac{3}{2}}}\right],
$$

where the two dots denote the second derivative with respect to $t$.

The configuration space of the above system is the first quadrant in the $(x, y)$ plane without the axes which correspond to the collision singularities of (4).

Note that if $\lambda_{12}=\lambda_{34}=0$ and $\alpha=1$, equations (4) describe a Kepler problem; Newtonian or Coulombian depending on the sign of $\lambda_{13}$. When $\lambda_{12}=\lambda_{34}=0$ and $\alpha<1$, equations (4) describe the Anisotropic Kepler problem, widely studied in $[3,5,9]$.

System (4) can be written in Hamiltonian form by taking $\mathbf{q}=(x, y)^{T}, M=$ $\operatorname{diag}\{2,2 \alpha\}$ and $\mathbf{p}=M \dot{\mathbf{q}}$. In these coordinates system (4) becomes

$$
\dot{\mathbf{q}}=\frac{\partial H}{\partial \mathbf{p}}, \quad \dot{\mathbf{p}}=-\frac{\partial H}{\partial \mathbf{q}},
$$

where

$$
H=\frac{1}{2} \mathbf{p}^{T} M^{-1} \mathbf{p}-U(\mathbf{q}) .
$$

and the potential $U$ is

$$
U=\frac{\lambda_{12}}{2 x}+\frac{4 \lambda_{13}}{\sqrt{x^{2}+y^{2}}}+\frac{\lambda_{34}}{2 y} .
$$

In short, the charged rhomboidal four-body problem can be formulated as the motion of a fictitious one particle of position $\mathbf{q}$ under the dynamics of the Hamiltonian system (5).

\subsection{McGehee Coordinates}

We introduce McGehee coordinates [15] in order to analyze the behavior of the orbits in a neighborhood of the total collision, in this way we define

$$
r=\sqrt{2\left(x^{2}+\alpha y^{2}\right)}, \quad \theta=\arctan \frac{\sqrt{\alpha} y}{x}, \quad v=r^{1 / 2} \dot{r}, \quad u=r^{3 / 2} \dot{\theta} .
$$


Since both $x$ and $y$ are positive we have that $\theta \in(0, \pi / 2) ; v$ and $u$ are the radial and tangential velocity of $\mathbf{q}$ scaled by the factor $r^{1 / 2}$. We also scale the time variable by $d t / d \tau=r^{3 / 2}$.

We note that $\theta=0$ corresponds to binary collision between the particles 3 and 4 , and $\theta=\pi / 2$ corresponds to binary collision between the particles 1 and 2 . On the other hand, $r=0$ corresponds to the total collision.

In McGehee coordinates system (4) becomes

$$
\begin{array}{ll}
\dot{r}=r v, & \dot{v}=u^{2}+\frac{v^{2}}{2}-U(\theta), \\
\dot{\theta}=u, & \dot{u}=-\frac{u v}{2}+U^{\prime}(\theta),
\end{array}
$$

where now the dot denotes the derivative with respect to $\tau$,

$$
U(\theta)=\sqrt{\frac{\alpha}{2}}\left[\frac{\lambda_{12}}{\sqrt{\alpha} \cos \theta}+\frac{8 \lambda_{13}}{\sqrt{\alpha \cos ^{2} \theta+\sin ^{2} \theta}}+\frac{\lambda_{34}}{\sin \theta}\right]
$$

and $U^{\prime}(\theta)$ means derivation with respect to the variable $\theta$. In the new variables the energy relation $H=h$ goes over to

$$
u^{2}+v^{2}=2 U(\theta)+2 h r .
$$

We observe that in equation (9) the potential $U$ depends only on the angular variable $\theta$, here we have used the fact that $U$ is a homogeneous function in the variables $x$ and $y$ with degree of homogeneity -1 . In Devaney [7] we can see that system (8) appears usually when we study the total collision manifold.

Since the original system (4) can be written in Hamiltonian form (5), in terms of a function which is quadratic in the momenta, we obtain that the system is reversible, property which persists when we introduce McGehee coordinates, in other words the system (8) possesses the symmetry $(r, v, \theta, u, \tau) \longrightarrow(r,-v, \theta,-u,-\tau)$.

The total collision manifold $\Lambda$ is characterized by

$$
\Lambda=\left\{(r, v, \theta, u): r=0, v^{2}+u^{2}=2 U(\theta), \quad \theta \in(0, \pi / 2)\right\} .
$$

Since $\dot{r}=0$ when $r=0$ in the first equation of (8), we have that $\Lambda$ is invariant under the flow; from the energy relation (10) we also have that $\Lambda$ is independent of the value of the constant energy $h$; i.e., each energy surface has the same total collision manifold $\Lambda$ in its boundary.

We note that by (11) the total collision manifold $\Lambda$ is not defined when $U(\theta)<0$ for all $\theta \in(0, \pi / 2)$. Clearly, the shape of the collision manifold is strongly related with the shape of the potential function $U(\theta)$. This function is analyzed in the following section.

\subsection{The total collision manifold}

In [1] there is a classification of all possible shapes of $U(\theta)$ with respect to the parameters when $\lambda_{12} \neq 0$ and $\lambda_{34} \neq 0$. In this paper we are interested just in the case on which the total collision manifold is compact. In this way we first fix the sign of the parameters in such a way that $\lambda_{12}<0, \lambda_{34}<0$ and $\lambda_{13}>0$. That is, we are supposing that we 
have repulsion between the particles 1 and 2, as well as between the particles 3 and 4 , and we have attraction between the particles 1 and 3, 1 and 4, 2 and 3, and 2 and 4 .

Let,

$$
\beta=\frac{\lambda_{12}}{\lambda_{34}}, \quad \delta=\frac{\lambda_{13}}{\lambda_{34}}, \quad \kappa=8 \delta(\alpha-1) .
$$

By the choice of the signs of the parameters we have that $\beta>0, \delta<0$ and $\kappa \geqslant 0$. The derivative of the potential function $U(\theta)$ given in (9) is

$$
U^{\prime}(\theta)=\sqrt{\frac{\alpha}{2}} \lambda_{34}\left[\frac{\beta \sin \theta}{\sqrt{\alpha} \cos ^{2} \theta}+\frac{\kappa \sin \theta \cos \theta}{\left(\alpha \cos ^{2} \theta+\sin ^{2} \theta\right)^{\frac{3}{2}}}-\frac{\cos \theta}{\sin ^{2} \theta}\right] .
$$

Let

$$
g(\theta)=\frac{\beta}{\sqrt{\alpha}}+\frac{\kappa}{\left(\alpha+\tan ^{2} \theta\right)^{\frac{3}{2}}}-\frac{1}{\tan ^{3} \theta},
$$

then

$$
U^{\prime}(\theta)=\sqrt{\frac{\alpha}{2}} \lambda_{34} \frac{\sin \theta}{\cos ^{2} \theta} g(\theta) .
$$

Therefore, the critical points of $U(\theta)$ for $\theta \in(0, \pi / 2)$ are the roots of the equation $g(\theta)=0$.

By straightforward computations we get

(i) $\lim _{\theta \rightarrow 0^{+}} g(\theta)=-\infty$, and $\lim _{\theta \rightarrow \pi / 2^{-}} g(\theta)=\beta \alpha^{-1 / 2}$.

(ii) If $g^{\prime}\left(\theta_{g}\right)=0$ then $\theta_{g}=\arctan \left[\alpha^{1 / 2}\left(\kappa^{2 / 5}-1\right)^{-1 / 2}\right]$, where $\kappa>1$.

(iii) $g\left(\theta_{g}\right)=\alpha^{-3 / 2}\left[\beta \alpha+\left(\kappa^{2 / 5}-1\right)^{5 / 2}\right]$.

(iv) The function $g$ has a unique zero $\theta_{0}$ in $(0, \pi / 2)$.

Using these properties of the function $g$, and since the potential function (9) satisfies that

$$
\lim _{\theta \rightarrow 0^{+}} U(\theta)=-\infty \quad \text { and } \quad \lim _{\theta \rightarrow \pi / 2^{-}} U(\theta)=-\infty,
$$

we obtain the next result.

Proposition 6 When $\lambda_{12}<0, \lambda_{34}<0$ and $\lambda_{13}>0$, the potential function $U(\theta)$ has exactly one critical point $\theta_{0}$ on the interval $(0, \pi / 2)$, which is a maximum.

Let $\theta_{0}$ be the critical point of $U$ given in Proposition 6 , we are interested in the possible sign of $U\left(\theta_{0}\right)$. Since $g\left(\theta_{0}\right)=0$, using (9) and (12), we get after some algebraic manipulations that

$$
U\left(\theta_{0}\right)=\sqrt{\frac{\alpha}{2}} \frac{1}{\cos ^{3} \theta_{0}}\left[\frac{8 \lambda_{13}}{\left(\alpha+\tan ^{2} \theta_{0}\right)^{\frac{3}{2}}}+\frac{\lambda_{34}}{\tan ^{3} \theta_{0}}\right] .
$$

From here, since $\theta_{0} \in(0, \pi / 2)$, the sign of $U\left(\theta_{0}\right)$ is the same that the sign of the expression between the brackets. After some computations we finally obtain the conditions on the parameters to get $U\left(\theta_{0}\right)>0$. We put them into the following result. 
Proposition 7 Let $\theta_{0}$ be the critical point of the potential $U(\theta)$, if $8 \delta+1<0$ and $\theta_{0}>\arctan \left[\alpha^{1 / 2}\left((-8 \delta)^{2 / 3}-1\right)^{-1 / 2}\right]$, then $U\left(\theta_{0}\right)>0$.

We observe that, if the parameters satisfy the hypotheses of Propositions 6 and 7 , then the potential function $U(\theta)$ is convex, it has a maximum $\theta_{0} \in(0, \pi / 2)$ with $U\left(\theta_{0}\right)>0, \lim _{\theta \rightarrow 0^{+}} U(\theta)=-\infty$, and $\lim _{\theta \rightarrow \pi / 2^{-}} U(\theta)=-\infty$ and consequently there are no binary collisions. Moreover, we can find $0<\theta_{1}<\theta_{0}<\theta_{2}<\pi / 2$ such that $U\left(\theta_{1}\right)=U\left(\theta_{2}\right)=0$ and $U(\theta)>0$ for all $\theta \in\left(\theta_{1}, \theta_{2}\right)$. In summary we have the next result.

Theorem 8 If $\lambda_{12}<0, \lambda_{34}<0$ and $\lambda_{13}>0,8 \delta+1<0$ and $\theta_{0}>$ $\arctan \left[\alpha^{1 / 2}\left((-8 \delta)^{2 / 3}-1\right)^{-1 / 2}\right]$, then the charged rhomboidal four body problem has a total collision manifold $\Lambda=\left\{(r, v, \theta, u): r=0, v^{2}+u^{2}=2 U(\theta), \theta \in\left[\theta_{1}, \theta_{2}\right]\right\}$ that is homeomorphic to a 2-dimensional sphere. Moreover there are no binary collisions between the particles, but there are total collisions.

\subsection{Equilibrium points}

In this section we compute the equilibrium points of system (8), which are strongly related with the critical points of the potential $U(\theta)$. Since the equilibrium points $\left(r_{0}, v_{0}, \theta_{0}, u_{0}\right)$ are zeros of the vector field given by (8) and satisfy the energy relation (10), we obtain

$$
r_{0}=0, \quad u_{0}=0, \quad U^{\prime}\left(\theta_{0}\right)=0, \quad v_{0}= \pm \sqrt{2 U\left(\theta_{0}\right)} .
$$

In the hypotheses of Theorem 8 we have that $U\left(\theta_{0}\right)>0$, so in this case the global flow given by (8) has two equilibrium points, both in $\Lambda$, given by (15) that, roughly speaking, they correspond to the northern and southern poles of $\Lambda$, respectively. We denote them by $e^{+}$and $e^{-}$according with $v_{0}>0$ or $v_{0}<0$.

Since the last three equations of system (8) do not depend on $r$ and the coordinate $r$ can be obtained from the energy relation (10), in order to describe the flow of (8) on a fixed energy level $H=h$, it is sufficient to describe the flow of the system formed by the last three equations of (8)

$$
\dot{v}=u^{2}+\frac{v^{2}}{2}-U(\theta), \quad \dot{\theta}=u, \quad \dot{u}=-\frac{u v}{2}+U^{\prime}(\theta) .
$$

We note that if the parameters satisfy the hypotheses of Theorem 8 , then the level of energy $E_{h}$ of (8) with $h<0$ is homeomorphic to the closed ball of $\mathbb{R}^{3}, \mathbb{D}^{3}=\{(u, \theta, v) \in$ $\left.\mathbb{R}^{3}: v^{2}+u^{2} \leqslant 2 U(\theta), \theta \in\left[\theta_{1}, \theta_{2}\right]\right\}$ with boundary $\mathbb{S}^{2}=\left\{(u, \theta, v) \in \mathbb{R}^{3}: v^{2}+u^{2}=\right.$ $\left.2 U(\theta), \theta \in\left[\theta_{1}, \theta_{2}\right]\right\}$. Moreover $\mathbb{S}^{2}$ is invariant under the flow of (16), see (10). From here on we take the coordinates of the points of $\mathbb{R}^{3}$ as $(u, \theta, v)$.

We linearize the vector field (16) at the equilibrium points $e^{+}=\left\{\left(0, \theta_{0}, \sqrt{2 U\left(\theta_{0}\right)}\right)\right\}$ and $e^{-}=\left\{\left(0, \theta_{0},-\sqrt{2 U\left(\theta_{0}\right)}\right)\right\}$. The eigenvalues at them are given by

$$
\mu_{1}=v_{0}, \quad \mu_{2,3}=\frac{-v_{0} \pm \sqrt{v_{0}^{2}+16 U^{\prime \prime}\left(\theta_{0}\right)}}{4}
$$


with eigenvectors

$$
v_{1}=(0,0,1), \quad v_{2,3}=\left(\frac{v_{0} \pm \sqrt{v_{0}^{2}+16 U^{\prime \prime}\left(\theta_{0}\right)}}{4}, 1,0\right),
$$

respectively. Note that the vectors $v_{2}$ and $v_{3}$ are tangent to $\mathbb{S}^{2}$.

We observe that if $v_{0} \neq 0$ and $U^{\prime \prime}\left(\theta_{0}\right) \neq 0$, then the two equilibrium points are hyperbolic. In this case we can compute the stable and the unstable invariant manifolds associated to these equilibrium points. We denote by $W_{P}^{s,(u)}$ the global stable (unstable) invariant manifold associated to the equilibrium point $P$. In the assumptions of Theorem 8, note that since $\theta_{0}$ is a maximum of $U\left(\theta_{0}\right)$, then $U^{\prime \prime}\left(\theta_{0}\right) \leqslant 0$.

A vector field $F$ is gradient like with respect to a function $g$ if all non-equilibrium solutions of $\dot{x}=F(x)$ are increasing with respect to $g$.

Theorem 9 Let $e^{+}$and $e^{-}$be the equilibrium points (15) for the flow given by (16), where $U\left(\theta_{0}\right)>0$ and $U^{\prime \prime}\left(\theta_{0}\right)<0$. We assume that the energy value is $h<0$.

(a) $W_{e^{+}}^{s}=\mathbb{S}^{2} \backslash\left\{e^{+}, e^{-}\right\}$where $\operatorname{dim} W_{e^{+}}^{s}=2$, and $W_{e^{+}}^{u}=\{(u, \theta, v): u=0, \theta=$

$\left.\theta_{0},-\sqrt{2 U\left(\theta_{0}\right)}<v<\sqrt{2 U\left(\theta_{0}\right)}\right\}=\Gamma$ where $\operatorname{dim} W_{e^{+}}^{u}=1$;

(b) $W_{e^{-}}^{s}=W_{e^{+}}^{u}$ and $W_{e^{-}}^{u}=W_{e^{+}}^{s}$.

Proof: From (16) the flow on $\mathbb{S}^{2}$ is given by

$$
\dot{v}=\frac{u^{2}}{2}, \quad \quad \dot{\theta}=u, \quad \dot{u}=-\frac{u v}{2}+U^{\prime}(\theta) .
$$

Since $\dot{v} \geqslant 0$ and it is not identically zero on any orbit of (18) different from $e^{+}$ and $e^{-}$, the vector field given by (18) is gradient like with respect to the coordinate $v$. Therefore, all the orbits on $\mathbb{S}^{2} \backslash\left\{e^{+}, e^{-}\right\}$have $\alpha$-limit $e^{-}$and $\omega$-limit $e^{+}$.

Observe that the segment $\Gamma$ is invariant under the flow of (16), it joins the equilibrium points $e^{+}$and $e^{-}$, and the flow on it goes in the decreasing direction of the $v$ axis. We note that the orbit $\Gamma$ is an ejection-collision homothetic orbit of (8), because $h<0$; i.e. it is an orbit that at any time form a central configuration.

By the Hartman's Theorem (see, for instance, [11]), and (17), the statements (a) and (b) follows.

\subsection{Symmetric periodic orbits}

Now we apply Theorem 3 to system (16). First we see that system (16) satisfies conditions $\left(C_{1}\right)-\left(C_{5}\right)$ of Section 2.

Assume that the parameters satisfy the hypotheses of Theorem 8. Then system (16) satisfies condition $\left(C_{1}\right)$ because $\mathbb{S}^{2}=\left\{(u, \theta, v) \in \mathbb{R}^{3}: v^{2}+u^{2}=2 U(\theta), \theta \in\left[\theta_{1}, \theta_{2}\right]\right\}$ is invariant under the flow of $(16)$. If $v_{0}^{2}+16 U^{\prime \prime}\left(\theta_{0}\right)<0$, then system (16) has two foci on $\mathbb{S}^{2}$, $e^{+}=\left\{\left(0, \theta_{0}, \sqrt{2 U\left(\theta_{0}\right)}\right)\right\}$ and $e^{-}=\left\{\left(0, \theta_{0},-\sqrt{2 U\left(\theta_{0}\right)}\right)\right\}$, so condition $\left(C_{2}\right)$ is satisfied. From Theorem 9, $W_{e^{+}}^{s}=W_{e^{-}}^{u}=\mathbb{S}^{2} \backslash\left\{e^{+}, e^{-}\right\}$and $W_{e^{+}}^{u}=W_{e^{-}}^{s}=\{(u, \theta, v): u=$ $\left.0, \theta=\theta_{0},-\sqrt{2 U\left(\theta_{0}\right)}<v<\sqrt{2 U\left(\theta_{0}\right)}\right\}=\Gamma$, thus conditions $\left(C_{3}\right)$ and $\left(C_{4}\right)$ are satisfied. 
Finally, since system (8) possesses the symmetry $(r, v, \theta, u, \tau) \longrightarrow(r,-v, \theta,-u,-\tau)$, system (16) is invariant under the symmetry $(u, \theta, v, \tau) \longrightarrow(-u, \theta,-v,-\tau)$, so condition $\left(C_{5}\right)$ is satisfied.

Let $\mathcal{L}$ be the heteroclinic loop formed by the equilibrium points $e^{+}$and $e^{-}$and their invariant manifolds $W_{e^{+}}^{s}=W_{e^{-}}^{u}$ and $W_{e^{+}}^{u}=W_{e^{-}}^{s}$. Applying Theorem 3 to system (16) we have that if the parameters satisfy the hypotheses of Theorem 8 and condition $v_{0}^{2}+16 U^{\prime \prime}\left(\theta_{0}\right)<0$, then for each $n \in \mathbb{N}$ system (16) has infinitely many periodic orbits near the heteroclinic loop $\mathcal{L}$ that cross exactly $2 n$ times the plane $v=0$ during a period. Clearly the periodic solutions of (16) give periodic solutions of (8) on the fixed energy level $E_{h}$. Therefore we have proved the following result.

Theorem 10 Assume the hypotheses of Theorem 8 and the condition $v_{0}^{2}+16 U^{\prime \prime}\left(\theta_{0}\right)<0$. For each $h<0$ and for each $n \in \mathbb{N}$, the charged rhomboidal four body problem (8) has infinitely many periodic orbits having fixed energy $h$ that pass close to total collision. Moreover these periodic orbits cross exactly $2 n$ times the plane $v=0$ during a period.

\section{Discussion section}

Using only geometrical and topological arguments we have provided sufficient conditions in order that a vector field in $\mathbb{R}^{3}$ has infinitely many periodic solutions near a heteroclinic loop $\mathcal{L}$ formed by a 2-dimensional sphere and an inner diameter of it. The use of geometrical arguments allows us to obtain the properties of the Poincaré map that are necessary in order to prove the existence of those periodic orbits without knowing the explicit analytic expression of the Poincaré map and without knowing the exact analytic expression of the vector field. Moreover the use of the mentioned geometrical arguments avoids the hard computations necessary for computing the image of the Poincaré map when the explicit analytic expression of the vector field is known.

We have also showed that the loop $\mathcal{L}$ appears in classical physics systems as the charged 4-body problem, and in systems which are being studied intensively by the mathematicians during these last years as the polynomials differential systems. Recently the study of the periodic orbits of polynomial vector fields in dimension larger than 2 is object of a great interest, see for instance [19, 20, 21] and the references quoted there.

Notice that we have only proved the existence of infinitely many periodic orbits near the loop $\mathcal{L}$, but we have not computed those periodic orbits explicitly. When the analytic expression of the vector field is known those periodic orbits could be computed analytically sometimes and numerically in general from the explicit analytic expression of the Poincaré map, but this was not the objective of this work.

The periodic orbits found here are not transversal as intersection of the stable and unstable manifolds so they are not related with the standard notion of chaos. Moreover these periodic orbits are not obtained from bifurcation because we do not need to move any parameter in order to obtain them. 


\section{Acknowledgements}

The first author is partially supported by the grant MCYT-Spain MTM2005-06098C02-01. The second author is partially supported by the grants MCYT-Spain MTM2005-06098-C02-01 and CIRIT-Spain 2005SGR 00550. The third author is partially supported by CONACYT-México Grant number 32167-E.

[1] F. Alfaro, and E. Perez-Chavela, The Rhomboidal Charged Four Body Problem, Hamiltonian Systems and Celestial Mechanics (HAMSYS 98), World Scientific Monograph Series in Mathematics, Vol. 6 (2000), 1-19.

[2] P. Atela and R. McLachlan, Global behavior of the charged isosceles three-body problem, Int. J. of Bifurcation and Chaos 4 (1994), 865-884.

[3] J. Casasayas and J. Llibre, Qualitative analysis of the anisotropic Kepler problem, Memoirs of the Amer. Math. Soc. 52, 1984.

[4] J. Casasayas and A. Nunes, A restricted charged four-body problem, Celestial Mechanics and Dynamical Astronomy 47 (1990), 245-266.

[5] G. Contopoulos and M. Harsoula, Stability and instability in the anisotropic Kepler problem, J. Phys. A 38 (2005), 8897-8920.

[6] R. Devaney, Blue sky catastrophes in reversible and Hamiltonian systems, Indiana Univ. Math. J. 26 (1977), 247-263.

[7] R. Devaney, Singularities in Classical Mechanical Systems, Ergodic Theory and Dynamical Systems I, Prog. Math. 10, Birkhäuser, 1981, pp 211-333.

[8] M. Fečkan, Blue sky catastrophes in weakly coupled chains of reversible oscillators, Discrete Contin. Dyn. Syst. Ser. B 3 (2003), 193-200.

[9] C. Gutzwiller, The anisotropic Kepler problem in two dimensions, J. Math. Physics 14 (1973), 139-152.

[10] J. Henrard, Proof of a conjecture of E. Strömgren, Celestial Mechanics 7 (1973), 449-457.

[11] M.W. Hirsch, C.C. Pugh and M. Shub, Invariant manifolds, Lecture Notes in Mathematics, Vol. 583, Springer-Verlag, Berlin-New York, 1977.

[12] I.D. Iliev, C. Li and J. Yu, Bifurcations of limit cycles from quadratic non-Hamiltonian systems with two centres and two unbounded heteroclinic loops, Nonlinearity 18 (2005), 305-330.

[13] Y.L. Jin and D.M. Zhu, Bifurcations of rough heteroclinic loops with three saddle points, Acta Math. Sin. 18 (2002), 199-208.

[14] J.S.W. Lamb and J.A.G. Roberts, Time-reversal symmetry in dynamical systems: a survey, Phys. D 112 (1998), 1-39.

[15] R. McGehee, Triple collision in the collinear three body problem, Inventions Math. 27 (1974), 191-227.

[16] E. Pérez-Chavela, D. Saari, A. Susin and Z. Yan, Central configuration in the charged three body problem, Contemporary Mathematics 198 (1996), 137-155.

[17] H. Poincaré, Les Mèthodes Nouvelles de la Mécanique Céleste, 3 Vols., Gauthier-Villars, Paris, 1892-1899, reprinted by Dover, New York, 1957.

[18] A. Vanderbauwhede, Heteroclinic cylces and periodic orbits in reversible systems, Ordinary and Delay Differential Equations, Pitman Reserach Notes in Mathematics Series 272, Pitman 1992, pp 250-253.

[19] H Żołądek, Limit cycles appearing after perturbation of certain multidimensional vector fields, J. Dynam. Differential Equations 13 (2001), 689-709.

[20] H Żołądek, Limit cycles for multidimensional vector fields. The elliptic case, J. Dynam. Control Systems 9 (2003), 265-310.

[21] H Żołądek, Limit cycles of three-dimensional polynomial vector fields, Nonlinearity 18 (2005), $175-209$. 